\title{
El buen humor como recurso didáctico en comunicación y lenguaje. Caso Facultad de Ciencias Humanísticas y Sociales, Universidad Técnica de Manabí 2017.
}

The good humor as a teaching resource in communication and language. Case Faculty of Humanities and Social, Technical University of Manabi 2017.

Dr. Edison Zambrano Cedeño Ph.D ${ }^{1}$, Mg. Juan Manuel Gómez Mieles ${ }^{2}$, Dra. Tatiana Quijano Velásquez ${ }^{3}$.

Recibido: 12-12-2017 / Revisado: 11-02-2018 Aceptado: 17-03-2018/ Publicado: 01-04-2018

\begin{abstract}
.
DOI: https://doi.org/10.33262/cienciadigital.v2i2.74

The present work proposes a new teaching methodology in higher education, using good humor as a didactic resource, understanding that it does not imply the execution of jokes or actions that provoke hilarity, on the contrary, it also implies a change of attitude part of the teacher, which goes from the greeting, the look, the daily deal with the student. Communication and Language was proposed by the CES, as a compulsory subject for the first levels of the courses redesigned in the national context, this, caused the arrival of a significant number of students to the classrooms, to a subject not so appreciated by them for the traditional way of imparting it, a syllabus was created based on the four macro skills: listening, speaking, reading and writing. A population of 840 students of first level of career was taken into account, whose sample was determined considering a margin of error of $5 \%$ and a confidence level of $95 \%$, for the calculation a certified web template was used. The method applied is descriptive, for which the techniques of documentary analysis and the
\end{abstract}

\footnotetext{
${ }^{1}$ Universidad Técnica de Manabí, Ecuador erzambrano@utm.edu.ec

${ }^{2}$ Universidad Técnica de Manabí, Ecuador,jmgomez@utm.edu.ec

${ }^{3}$ Universidad Técnica de Manabí, Ecuador, nquijano@utm.edu.ec
} 
survey were used. It concludes with the theory that applying good humor as a didactic resource is an effective aid to achieve significant learning in the student.

Keywords: Communication and Language, Psychopedagogical, Meaningful Learning, Good Humor and Education, Good Humor and Higher Education

\section{Resumen.}

El presente trabajo propone una nueva metodología de enseñanza en la educación superior, utilizando el buen humor como un recurso didáctico, entendiéndose que, éste no precisamente implica la ejecución de chistes o acciones que provoquen hilaridad, al contrario, también supone un cambio de actitud por parte del docente, que va desde el saludo, la mirada, el trato cotidiano con el estudiante. Comunicación y Lenguaje fue propuesta desde el CES, como materia obligatoria para los primeros niveles de las carreas rediseñadas en el contexto nacional, esto, provocó la llegada de un número importante de estudiantes hasta las aulas, a una asignatura no tan apreciada por ellos por la forma tradicional de impartirla, se creó un sílabo basado en las cuatro macro destrezas: escuchar, hablar, leer y escribir. Se tomó en cuenta una población de 840 estudiantes, de primer nivel de carrera, cuya muestra se determinó considerando un margen de error del 5\% y un nivel de confianza del 95\%, para el cálculo se utilizó una plantilla web certificada. El método aplicado es el descriptivo, para lo cual se utilizaron las técnicas de análisis documental, y la encuesta. Se concluye con la teoría de que el aplicar el buen humor como un recurso didáctico es una ayuda efectiva para lograr aprendizajes significativos en el estudiante.

Palabras Claves: comunicación y lenguaje, psicopedagógico, aprendizaje significativo, buen humor y educación, buen humor y educación superior

\section{Introducción.}

Desde septiembre del 2014, en el Ecuador se dispuso desde el Consejo de Educación Superior (CES), el rediseño de todas las carreras que ofertaban las universidades e institutos de educación superior del país, con el afán de mejorar la calidad educativa y tener estándares altos de desempeño, un rediseño que no sólo abarcaba la revisión del currículo, sino también de la estructura que sostenía su contenido.

Dentro de la propuesta, se planteó una malla genérica, en la cual constaba de manera obligatoria la asignatura de Comunicación y Lenguaje en el primer nivel de carrera, lo que provocó que, en el 2017, la Universidad Técnica de Manabí y el departamento de Ciencias de la Información, se crearan 48 paralelos para albergar a 2238 estudiantes de primer nivel, de todas las carreras hasta ese momento rediseñadas, que eran 29. 
Frente a esta realidad y, conociendo que la gran mayoría de carreras son técnicas, este estudio se orientó hacia la investigación de cómo el buen humor ayudaría en la impartición de la materia a fin de producir una estrategia metodológica y didáctica para hacerla más amena y sobre todo productiva en cuanto a los conocimientos.

Dentro del sílabo se trabajó con las macro destrezas que los estudiantes en el hasta el bachillerato han desarrollado, estas son Escuchar, Hablar, Leer y Escribir, en las que se insertaron actividades específicas para cada unidad de contenidos. En este orden de cosas, este trabajo investigativo se convierte en una herramienta para los docentes universitarios en tanto y cuanto quieran cambiar su manera tradicional de trabajar en el aula de clases.

Por otra parte, es una respuesta a las frecuentes dificultades que enfrentan muchos docentes a la hora de impartir esta clase de asignaturas, que perse, son eminentemente teóricas y más, con estudiantes que han escogido carreras técnicas, en las cuales no esperan recibir esta clase de asignaturas.

Es por ello que en esta investigación tiene como objetivo principal demostrar que una clase impartida con buen humor, es generadora de conocimientos, potencia las actividades áulicas, enriquece y desarrolla las destrezas que tienen los estudiantes en sus diferentes ámbitos.

El sentido del humor es una capacidad única del ser humano. Todas las culturas lo han valorado y le atribuyen numerosos beneficios psicológicos, tales como, estados de alegría, bienestar y satisfacción, menor estrés y depresión. Se le asignan también, beneficios físicos como tolerancia al dolor, activación del sistema inmune, cardiovascular y respiratorio, y sociales, mejora de la productividad, la motivación, la comunicación, el orden y armonía sociales.

Para lograr este trabajo investigativo se aplicó una metodología descriptiva con enfoque cuali- cuantitativo y se tomó en cuenta una población de 840 estudiantes del primer nivel de carrera y la muestra fue calculada con fórmula estadística con un nivel de confianza del 95\%, a quienes se les aplicó encuesta.

\section{Marco teórico referencial.}

\section{El buen humor como parte del bienestar del ser humano.}

Bustamante, et.al (2014) afirma que el bienestar psicológico correlaciona con el sentido del humor e implica tres variables afectivas como la Alegría, la Seriedad y el Mal Humor, 
como bases temperamentales del mismo y que se consideran disposiciones estables (rasgos) o circunstanciales (estados)

Siendo un tema de gran expectativa y sumamente nuevo, el estudio del humor guarda una gran riqueza y amplias posibilidades por su escasa exploración científica. Las investigaciones actuales suscitan amplios caminos de análisis, aplicaciones y usos terapéuticos, educacionales y sociales. El futuro inmediato en los estudios sobre el humor se enfoca hacia modelos educacionales por el hecho de estar en contacto directo con el proceso de desarrollo del ser humano

El estudiante universitario tiene características especiales, generalmente es mayor de edad, algunos tienen un hogar con una pareja establecida, otros son solteros pero se pagan sus estudios universitarios, hay madres solteras, teniendo este panorama, realmente y sabiendo que el estudiante es el protagonista del proceso de enseñanza-aprendizaje, se incorporan metodologías activas al proceso docente. Aprendizaje por construcción o significativo, aprendizaje social, aprendizaje colaborativo o cooperativo y aprendizaje basado en proyectos son algunas de las nuevas metodologías que se manejan en los foros de innovación educativo, el aprendizaje heurístico, basado en el descubrimiento, dentro de todos ellos el buen humor juega un papel importante dentro de su desarrollo.

López et. al (2009), afirma que tener sentido del humor significa intentar responder con una actitud positiva ante los retos o situaciones difíciles que nos encontramos en el día a día. El humor permite enfrentarse a una situación difícil sin dejarse secuestrar por las emociones negativas. Ayuda a crear ambientes más relajados y favorables para la toma de decisiones y la solución de conflictos; y protege, en cierta medida, contra el estrés.

Fernández (1999) expresa que el humor tiene la siguiente función pedagógica: "Se agilizan y enriquecen los procesos de enseñanza y aprendizaje. En los procesos de enseñanza el humor es un componente que introduce nuevas variables en la elaboración y transmisión de información. En los procesos de aprendizaje el sentido del humor ayuda a mejorar la calidad y el rendimiento de las tareas. Genera una actitud positiva ante la ocupación. Ayuda a desarrollar la memoria y los procesos cognitivos. Favorece la atención. Aumenta la motivación. Ayuda a mejorar la relación educadora-educando".

El humor es una herramienta que nos sirve para edificar mundos más confortables, embelleciendo nuestras vidas, inmunizándonos y actuando como calmante. Dicen que la risa y la diosa del amor siempre fueron amigas. En definitiva, puede ser un foco que arroje luz sobre nuestra propia vida y un arma que dinamite convenciones y transgreda normas (Jiménez y Fernández 2006) 


\section{El humor en la enseñanza.}

Ese contacto visual, esa charla sobre el equipo de fútbol preferido, sobre un caso en particular, esa palmada en la espalda, eso es buen humor, lo que va a propiciar el desarrollo de las capacidades de cada uno de sus estudiantes en el aula de clases, esto sumado a actividades grupales, dramatizaciones y trabajos de creación colectiva.

Muchas han sido las veces en la que los docentes han puesto su interés en las actividades áulicas que se imparten con humor, coincidiendo en los beneficios que presta el buen humor dentro de la enseñanza. Así por ejemplo Rodríguez (2008), afirma que el humor como estrategia pedagógica es una propuesta para romper los paradigmas tradicionales de la educación, y crear un ambiente agradable de aprendizaje y comprensión de los temas propios de la educación formal.

Algunos focalizan el uso del humor en la desdramatización del vínculo entre el estudiante y la asignatura. Otros intentan utilizarlo para afianzar el vínculo entre el docente y el estudiante y también hay autores que destacan la importancia del humor para mejorar las relaciones entre compañeros. (Kanovich 2008)

\section{El buen humor en el contexto universitario.}

La academia, concebida en su espectro como la generadora de investigación, gestión, resultados, y todas ellas integralmente unidas, para que produzca los efectos necesarios para la sociedad en la que se desenvuelve, es donde se encuentra el pensamiento crítico, el pensamiento que parte de una reflexión severa, compleja, esto es formar para el cuestionamiento de las situaciones que los rodean y de los fenómenos naturales y sociales; promover una mentalidad abierta y transformadora de la realidad, formar sujetos que desconfíen de la pretendida neutralidad de la información; desplegar un escepticismo reflexivo; desarrollar habilidades de pensamiento superior como capacidad interrogativa, destrezas dialógicas, exploración de alternativas, resolución de problemas complejos; promover el pensamiento creativo y la capacidad de valoración ética, estética, social, intelectual y política.

Con todas estas premisas como perfil de egreso genérico de una universidad, todavía existen docentes que no entran en este mundo, al contrario, siguen pensando que los contenidos tienen mucha más importancia que la valoración crítica. 


\section{Marco metodológico.}

La metodología aplicada para este trabajo fue cuali-cuantitativa, de tipo descriptivo y se aplicó análisis documental, a partir de la información recopilada por medio del estudio y análisis pormenorizado de numerosos textos referentes al tema que está en estudio.

La investigación se centró en el caso específico de la Facultad de Ciencias Humanísticas y Sociales de la UTM, dentro del departamento de Ciencias de la Información, donde académicamente está ubicada la asignatura de Comunicación y Lenguaje y se trabajó con 16 paralelos del primer nivel de carrera, obteniendo mediante una plantilla web certificada, una muestra de 264 estudiantes, a quienes se les aplicó una encuesta de 12 preguntas, con un nivel de confianza del 95\% y con un margen de error del 5\%.

\section{Análisis de resultados.}

Los resultados que se tomaron en cuenta fueron los siguientes:

Se realizó un cuestionario que constó de 12 ítems:

Tabla 1: Conocimiento acerca del buen humor

\begin{tabular}{lccc}
\hline \multirow{2}{*}{ LITERAL } & \multirow{2}{*}{ ALTERNATIVAS } & \multicolumn{2}{c}{ ESTUDIANTES } \\
& & F & $\%$ \\
\hline a. & Mucho. & 0 & $0 \%$ \\
b. & No mucho. & 66 & $25 \%$ \\
c. & Poco. & 132 & $50 \%$ \\
d. & Casi nada. & 61 & $23 \%$ \\
e. & Nada. & 5 & $2 \%$ \\
& TOTAL & 264 & $100 \%$ \\
\hline
\end{tabular}

Elaboración: Grupo de Investigación.

Fuente: Estudiantes de primer nivel de carrera Universidad Técnica de Manabí.

El estudiante tiene muy poco conocimiento del buen humor, lo asocian simplemente con chistes, bromas y no se dan cuenta que va más allá, depende del trato, de la comunicación, tal como lo afirma Leiva y Rozas el humor nace de una experiencia radical del ser humano: el sentir los límites de lo individual. El contexto universal del humor es lo inmediato y sus límites. Por lo tanto, el humor implica vernos viviendo.

El buen humor es una matriz de acción que nos mueve a una perspectiva abierta para entendernos. En ella está implícito los siguientes factores: aceptar las limitaciones, corregir errores, abrirnos a la experiencia, superar los límites que nos imponen, y superar los límites que nos imponemos, y, principalmente, escapar del sufrimiento, sin embargo, se pude notar 
que el buen humor por desconocimiento no se utiliza ni se transmite como herramienta en la que el docente y el estudiante puedan mejorar dentro de sus actividades.

Tabla 2: Como debe ser una clase con buen humor.

\begin{tabular}{|c|c|c|c|}
\hline \multirow{2}{*}{ LITERAL } & \multirow{2}{*}{ ALTERNATIVAS } & \multicolumn{2}{|c|}{ ESTUDIANTES } \\
\hline & & $\mathbf{F}$ & $\%$ \\
\hline a. & Potenciadora del aprendizaje. & 53 & $20 \%$ \\
\hline b. & Interesante. & 74 & $28 \%$ \\
\hline c. & Motivadora e inspiradora. & 63 & $24 \%$ \\
\hline d. & Desestresante. & 63 & $24 \%$ \\
\hline e. & Normal. & 11 & $4 \%$ \\
\hline & TOTAL & 264 & $100 \%$ \\
\hline
\end{tabular}

Elaboración: Los autores.

Fuente: expertos en ingeniería de software. Centro de cómputo Universidad Técnica de Manabí.

La educación universitaria es uno de los aspectos más importantes del ser humano, por lo cual debe ser un proceso de formación enriquecedor en todos sus aspectos, es allí donde recae la responsabilidad del docente como trasmisor de sabios conocimientos, es él, quien debe de buscar las herramientas, técnicas e instrumentos necesarios con el fin de que la clase sea amena y desarrollada con éxito, he aquí donde el buen humor cumple su papel satisfactorio dentro de la formación profesional.

Como lo sustenta Liébana, el buen sentido del humor puede considerarse como un rasgo positivo ya que hace que la persona que lo tiene pueda experimentar la risa y el placer subjetivo asociado a ella, así como los beneficios psico-físicos vinculados a esta, más frecuentemente.

Por ello es indispensable dejar claro que dentro del salón de clases una de las fortalezas que deben de formar parte es el sentido del humor, ya que forma parte de la psicología positiva siendo así una herramienta fácil de adquirir. 
Tabla 3: Trato del docente con buen humor.

\begin{tabular}{llcc}
\hline \multirow{2}{*}{ LITERAL } & \multirow{2}{*}{ ALTERNATIVAS } & \multicolumn{2}{c}{ ESTUDIANTES } \\
& & F & \% \\
\hline a. & Amable y cordial. & 63 & $24 \%$ \\
b. & Motivador. & 103 & $39 \%$ \\
c. & Empático. & 13 & $5 \%$ \\
d. & Comprensivo. & 77 & $29 \%$ \\
e. & Común y corriente. & 8 & $3 \%$ \\
& TOTAL & 264 & $100 \%$ \\
\hline
\end{tabular}

Elaboración: Los autores

Fuente: expertos en ingeniería de software. Centro de cómputo Universidad Técnica de Manabí

Es importante tener claro que la motivación es uno de los aspectos fundamentales para incentivar el cumplimiento de objetivos planteados o propuestos, por ello dentro de la educación universitaria es indispensable que los docentes estén motivados a enseñar y de la misma forma trasmitan la motivación necesaria a los futuros profesionales, con el fin de que pueda tener éxito en su función laboral.

En el primer nivel de carrera los estudiantes llegan con muchas expectativas, ilusiones, sueños, en su gran mayoría pensando que al terminar la universidad tienen asegurado un futuro promisorio. Entonces ese primer contacto con el mundo universitario, debe ser enamoradizo, convincente, es ahí donde el buen humor tiene asidero como técnica didáctica para lograr los objetivos deseados. La motivación por parte del docente debe ser un punto de partida esencial.

Como lo sustenta Pila Jorge, Malow determina a la motivación dentro del aula de clases como "un concepto abstracto, un concepto hipotético que existe para poder explicar la razón por la cual la gente se comporta y piensa de la forma que lo hace. La motivación está relacionada con uno de los aspectos más básicos de la mente humana y esta tiene un rol determinante en el éxito o fracaso en cualquier situación de aprendizaje" 
Tabla 4: Debe existir buen humor en la universidad

\begin{tabular}{llcc}
\hline \multirow{2}{*}{ LITERAL } & \multirow{2}{*}{ ALTERNATIVAS } & \multicolumn{2}{c}{ ESTUDIANTES } \\
& & F & \% \\
\hline a. & $\mathrm{Si}$. & 198 & $75 \%$ \\
b. & No. & 66 & $25 \%$ \\
& TOTAL & 264 & $100 \%$ \\
\hline
\end{tabular}

\section{Elaboración: Los autores}

Fuente: expertos en ingeniería de software. Centro de cómputo Universidad Técnica de Manabí

En el sistema educativo es indispensable que exista el buen humor, y más aun a nivel universitario donde los estudiantes son personas adultas y con una mentalidad mucho más abierta.

Muchos de ellos llegan dejando sus hijos/as con la vecina, con algún pariente, con todos los riesgos que esto implica; algunos/as llegan con problemas fuertes y complejos; en este caso los docentes deben de tener en cuenta que en el ambiente universitario si debe existir el buen humor que les permita a los futuros profesionales crear una ideología científica a través de esa parte humorística que ayudará a su rendimiento en el desempeño profesional, aunque para, Dziegielewski, Jacinto, Laudadio y Legg-Rodríguez afirman que incluso el humor es concebido como poco profesional. No obstante, el estudio del fenómeno denominado humor es abordado desde muy diversas disciplinas: antropología, sociología, lingüística, psicología, fisiología, medicina, filosofía o educación y constituye, por lo tanto, un campo interdisciplinar.

Tabla 5: Docentes que utilizan buen humor.

\begin{tabular}{|c|c|c|c|}
\hline \multirow{2}{*}{ LITERAL } & \multirow{2}{*}{ ALTERNATIVAS } & \multicolumn{2}{|c|}{ ESTUDIANTES } \\
\hline & & $\mathbf{F}$ & $\%$ \\
\hline a. & 1 & 0 & $0 \%$ \\
\hline b. & 0,75 & 0 & $0 \%$ \\
\hline c. & 0,5 & 108 & $41 \%$ \\
\hline d. & 0,25 & 95 & $36 \%$ \\
\hline \multirow[t]{2}{*}{ e. } & Ninguno. & 61 & $23 \%$ \\
\hline & TOTAL & 264 & $100 \%$ \\
\hline
\end{tabular}

Elaboración: Los autores.

Fuente: expertos en ingeniería de software. Centro de cómputo Universidad Técnica de Manabí 
El buen humor está demostrando a lo largo de vida educativa, que es un sentimiento que permite estar bien consigo mismo y transmitir a su vez a las personas que están a su alrededor. Dentro de estas aseveraciones, el aula de clases universitaria es para los docentes un espacio donde pueden convertir el mundo de los estudiantes con buen sentido del humor $\mathrm{y}$ algunos han logrado que ellos puedan comprender y entender cada una de las actividades, de esta manera los futuros profesionales se destacan desde su proceso educativo y alcanzan el éxito en la adquisición de experiencia laboral.

Escalona (2000), en su trabajo sobre "El sentido del buen humor en la educación", comenta: La persona con sentido del humor es, en las relaciones humanas, comprensiva. Entiende, "tiene sentido" del humor, es decir, comprende lo que pasa a sus semejantes y a él mismo. Comprende que no es tan fácil mostrarnos tan buenos como somos debido al 'humor", a nuestro estado de salud espiritual.

\section{Conclusiones}

- Los estudiantes universitarios de primer nivel de carrera tienen poco conocimiento acerca de lo que realmente es el buen humor, además destacan que se aplica en mínimas oportunidades como una técnica para enriquecer los conocimientos.

- El buen humor es una técnica didáctica que, aplicada en la asignatura de Comunicación y Lenguaje, permite a los estudiantes universitarios adquirir aprendizajes significativos, por ello consideran que debe utilizarse siempre o casi siempre en una clase y proponen que sea en todas las áreas.

- El ambiente de aula universitario con buen humor se torna ameno, divertido, motivador, des estresante y eso provoca al final que mejore de manera significativa la calidad de los aprendizajes.

- Los docentes universitarios tienen poco sentido del buen humor y piensan que al hacer una clase con esta técnica perderán respeto ante sus estudiantes. Sin embargo, están conscientes que al ser amables, empáticos, comprensivos con sus estudiantes y aplicando dinámicas va a obtener siempre mayor motivación en las actividades educativas.

- Las clases de Comunicación y Lenguaje, impartidas con buen humor han llegado siempre a la comprensión total, mejorando el nivel de comunicación, y cumplimiento de las actividades planteadas en el sílabo. 


\section{Referencias bibliográficas.}

Bustamante, J., \& Salavera, C., \& Antoñanzas, J., \& Chueca, J., \& Teruel, P., \& Carrón, J., \& Larrosa, S., \& Bericat, C. (2014). LA PERSONALIDAD EFICAZ EN EL UNIVERSITARIO Y EL USO DEL BUEN HUMOR. International Journal of Developmental and Educational Psychology, 1 (1), 467-475.

López Martínez, O., \& Sevilla Moreno, A., \& Ruiz Esteban, C. (2009). ¿POR QUÉ LA CREATIVIDAD $Y$ EL SENTIDO DEL HUMOR EN LA EDUCACIÓN?. International Journal of Developmental and Educational Psychology, 2 (1), 281-289.

Fernández Solís, J.D. (1999) "Hacia una pedagogía del humor”, Revista Naque: teatro, expresión, educación, n. ${ }^{\circ}$ 10, Ciudad Rea

Jiménez García, E., \& Fernández Vallejo, G. (2006). HUMOR Y CREATIVIDAD: UNA EXPERIENCIA EDUCATIVA.International Journal of Developmental and Educational Psychology, 2 (1), 441-449.

Rodríguez C. (2008) El buen humor como estrategia pedagógica, Tesis de maestria en docencia. Manizales, Colombia.

Kanovich, S. (2008). El uso del humor en la enseñanza universitaria. Cuadernos de Investigación Educativa, 2 (15), 71-90.

Leiva, T \& Rozas, X. (2010). Proyecto de Tesis: "El sentido del humor en la práctica docente, como facilitador de aprendizajes significativos en los estudiantes, en las clases de Historia y Ciencias Sociales”, pág. 21. Santiago.

Liébana, C. (2014). Tesis Doctoral: "El sentido del humor en el aula: diseño, aplicación y evaluación de un programa de intervención” pág. 26-27-31-32. Valladolid.

Malow, H. (2010). sustentado por Pila Chipugsi Jorge Edison, (2012) Trabajo de grado: "La motivación como estrategia de aprendizaje en el desarrollo de competencias comunicativas” pág. 6, Guayaquil-Ecuador.

Dziegielewski, S. \& y otros.(2003). Humor: An Essential Communication Tool in Therapy. International Journal of Mental Health, 32, (3), 74-90.

Escalona I (2010). Trabajo de fin de grado: "El sentido del humor en la educación”. 1112. Valladolid. 


\section{Para citar el artículo indexado.}

Zambrano E., Gómez J. \& Quijano T. (2018). El buen humor como recurso didáctico en comunicación y lenguaje. Caso facultad de ciencias humanísticas y sociales, universidad técnica de Manabí. 2017. Revista electrónica Ciencia Digital 2(2), 79-90. Recuperado desde:

http://cienciadigital.org/revistacienciadigital2/index.php/CienciaDigital/article/view/74/69

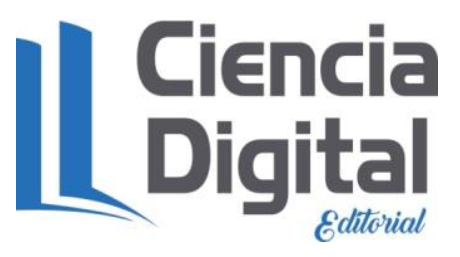

El artículo que se publica es de exclusiva responsabilidad de los autores y no necesariamente reflejan el pensamiento de la Revista Ciencia Digital.

El articulo queda en propiedad de la revista y, por tanto, su publicación parcial y/o total en otro medio tiene que ser autorizado por el director de la Revista Ciencia Digital.
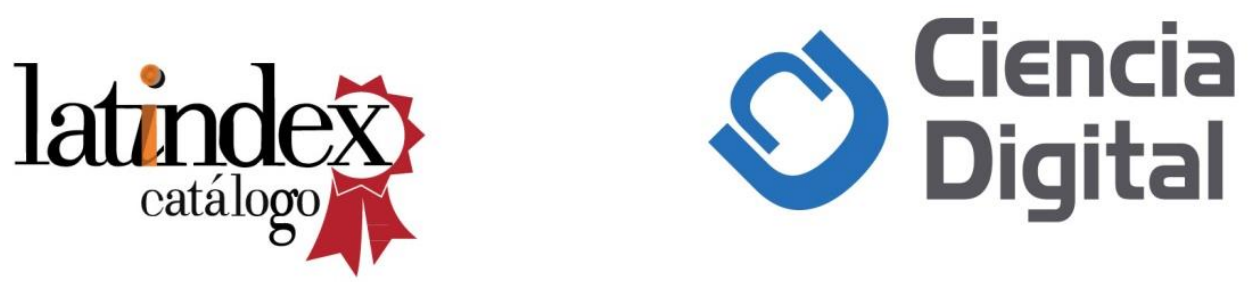